\title{
Accidents in platoons of vehicles
}

\author{
Cécile Appert-Rolland ${ }^{1}$ and Ludger Santen ${ }^{2}$ \\ 1 Université de Paris-Sud, Laboratoire de Physique Théorique, Bâtiment 210, \\ F-91405 Orsay Cedex, France - appert@th.u-psud.fr \\ 2 Universität des Saarlandes, Fachrichtung Theoretische Physik, \\ D-66041 Saarbrücken, Germany - santen@lusi.uni-sb.de
}

\begin{abstract}
In dense vehicular traffic cars often drive at close distances, they form clusters or platoons. Within these platoons, time headways are observed, which are often even shorter than the reaction time of the drivers - a situation which is potentially dangerous.

Here we propose a simple dynamical model for a platoon undergoing emergency braking, which takes into account the individual variations of the reaction time and braking capacities. We apply the model to real platoons, i.e. platoons which have been identified in large sets of single-vehicle traffic data. We use our results in order to compare the impact of different possible regulations (speed limit, minimum headway).
\end{abstract}

\section{Introduction}

One of the remarkable empirical features of highway traffic is the fact that vehicles frequently undergo the security distance. Although the recommended security distance amounts to $1.8 \mathrm{sec}$ in Germany and the observed time headway distribution has in general a maximum around 1. sec, one frequently observes time headways far below one second [1]. These extremely short time headways have important consequences concerning the structure, performance and security of vehicular traffic. In this work we focus on the security aspects of dense highway traffic. The search for causes of accidents in vehicular traffic has attracted broad scientific interest. Studies on this subject include the identification of dangerous situations in model generated configurations. Although many interesting results have been obtained using this model-based approach it is not obvious how these results depend on the particular model. Therefore we try to minimize the modeling part throughout our analysis by taking empirical data of highway traffic in combination with a simple braking model. The usage of empirical data implies that we consider properly all relevant correlations between time-headways and velocities. This procedure should lead to more significant results than obtained in previous studies. As we are interested in the risk related to the structure of traffic flows we analyze a typical situation where a chain reaction of brakings leads to an accident. This will happen in platoons, i.e. clusters of vehicles driving at short distances.

Let us consider a platoon including $N$ vehicles $(i=0 \ldots N-1)$. Each vehicle has a velocity $v_{i}$, a reaction time $\tau_{i}$ and a braking capacity $a_{i}^{*}$. The time-headway $t_{i}^{h}$ is defined here as the temporal distance between the rear end of the preceeding vehicle $i-1$ and the front end of vehicle $i$. Now we assume that one car in this 
platoon is braking with deceleration $a_{0}$. If time headways are small enough, the following cars have to brake as well in order to avoid collision. More precisely, if $t_{i}^{h}<\tau_{i}$, vehicle $i$ has to brake harder than vehicle $i-1$. If this is true for several vehicles in a row it finally may happen that the required deceleration exceeds the braking capacity of the car. In this case a collision occurs.

Apart from this mechanism it is possible that a driver didn't even start to brake before colliding with the preceeding car if his reaction time is too long (or the time headway too short). As a result, the accident probability is increasing with the position in the platoon. This well-known effect has already been studied in ideal platoons e.g. having constant initial time headways, in particular to study the impact of various cruise control devices [2]. In order to improve the realism of the approach some authors have introduced probability distributions for the reaction times or braking capacities [3]. Although this approach recognizes the variability of time headways, speeds and reaction times it disregards the fact that e.g. velocity-velocity or velocity-time headway correlations are non-negligible in dense vehicular traffic. Correlations can be included by directly identifying the platoons in the empirical data sets (see section 3 for details).

In the scenario that we consider, cars undergo emergency braking. We thus have to introduce a dynamical model for such emergency braking, which we keep as simple as possible: Braking of car $i$ triggers the braking of the following car $i+1$, which starts to brake after a reaction time $\tau_{i+1}$. Then the car slows down with the weakest constant deceleration sufficient to avoid accidents, until it stops. This choice of a constant deceleration law is consistent with deceleration records for emergency braking on tracks (see e.g. [4]), although other empirical studies indicate that unexpected braking on real roads with non professional drivers is rather described by a two step process [5]. However, so far there exists only a small number of empirical studies on this subject, such that it is difficult to single out one of the two possibilities. Therefore we have chosen the constant deceleration law for simplicity.

The event that triggers the chain of emergency brakings is the sudden braking of an initiating car with a constant deceleration $a_{0}$. We chose a modest value for $a_{0}$ (between 3 to $5 \mathrm{~m} / \mathrm{s}^{2}$ ), well below the average maximum braking capacity. The choice of the model implies that we have to assign two parameters to each vehicle, which are not included in the data set: the reaction time of the drivers and the maximum braking capacities. These are taken from probability distributions, which rely on the outcome of e.g. car-following experiments. There is a consensus to take a log-normal distribution for the reaction times. The parameters of this distribution differ depending whether the driver expects the considered event or not. In a platoon, drivers expect to have to adjust their velocity to the preceeding cars very often, and thus they react quite rapidly if a weak acceleration is required. However, they do not expect an emergency braking, and it is a well known fact that, due to their fear of rear-end collision with the following car, drivers hesitate to brake too hard. Therefore we used a distribution of reaction times which corresponds to unexpected events. The log-normal distribution for the reaction times has to be cut off beyond a certain 
value, that we took equal to $2 \mathrm{~s}$ following [4]. Still, some authors [6] claim that some much longer reaction times may be observed in real traffic, and the effect of these will be considered in future work. It is however reasonable to assume that in platoons, where people expect to have to adapt their speed all the time, very long reaction times would be exceptional.

In this paper, we took for the braking capacity distribution a Gaussian centered around $7 \mathrm{~m} / \mathrm{s}^{2}$, and truncated below 6 and above $8 \mathrm{~m} / \mathrm{s}^{2}$, except when stated otherwise.

We stress the fact that the braking capacity and reaction time distribution are the only model parameters that can not be directly taken from the single vehicle data. Contrary the structure of the platoons is directly accessible and does not depend on model parameters of any kind. In order to obtain representative values of the accident probabilities by means of numerical simulations we have considered 500 to 1000 realizations of the probability distributions.

\section{Criterium for accidents}

A first estimate for the number of accidents in a platoon can be obtained simply by comparing the final positions of the cars after braking. Obviously an accident must have occurred if the order of cars is exchanged compared to the initial positions. The corresponding criterium on deceleration requirements reads

$$
\frac{1}{a_{i}} \leq\left(\frac{v_{i-1}^{0}}{v_{i}^{0}}\right)^{2} \frac{1}{a_{i-1}}+\frac{2\left(t_{i}^{h}-\tau_{i}\right)}{v_{i}^{0}} .
$$

However, some of the accidents may not be identified by means of the final positions. Figure 1 shows such an example, where the trajectories of two cars intersect, although the final positions do not indicate the occurrence of an accident. In order to consider such kinds of accidents as well, one has to introduce an alternative criterium in a certain range of parameters [7]

$$
\frac{1}{a_{i}} \leq \frac{2\left(v_{i-1}^{0}-v_{i}^{0}\right) \tau_{i}+2 v_{i}^{0} t_{i}^{h}-a_{i-1} \tau_{i}^{2}}{2 a_{i-1} v_{i}^{0} t_{i}^{h}+\left(v_{i-1}^{0}-v_{i}^{0}\right)^{2}} .
$$

In our study on, which relies on a large set of real data, we found that about $15 \%$ of the collisions, i.e. a non-negligible fraction, are identified by means of (2).

\section{Description of the data set}

The data have been collected between March, 30, 2000 and May, 16, 2000 on the German highway A3 between the junction Duisburg-Wedau and the highwayintersection Kreuz-Breitscheid. The traffic stream characteristics at this location have been established by magnetic loops, one for each lane. The chosen location is well apart from on- and off-ramps or intersections, such that the data set should represent the bulk properties of real vehicular traffic. It also important to note 


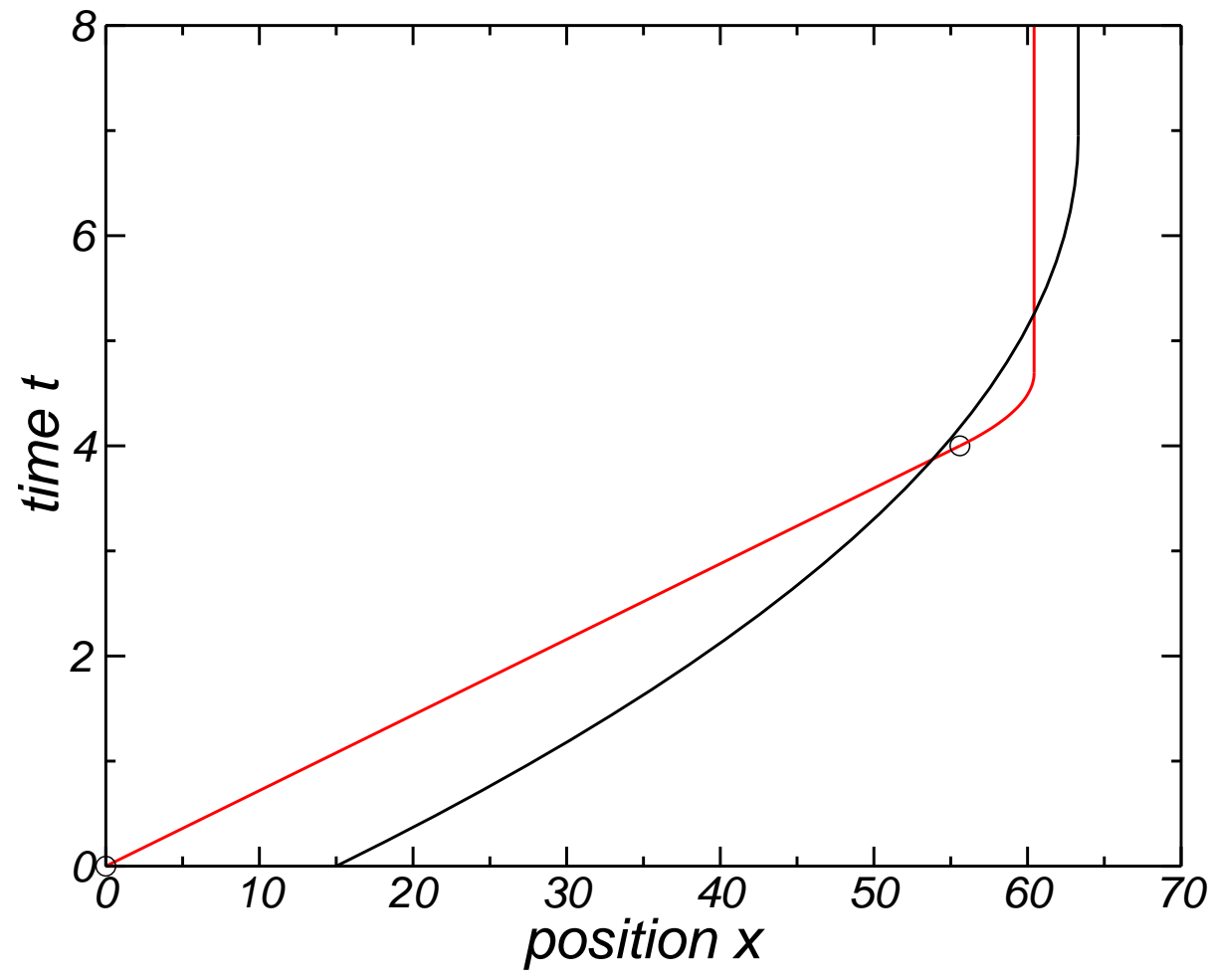

Fig. 1. Trajectories of two successive cars. The circle indicates the end of the reaction time for the 2 nd driver. When two cars are very close, but the second car has a deceleration capacity much stronger than the first car, it is possible to have an intersection between the trajectories that would not be detected if one looked only on the final positions.

that there is no speed limit applied at this section of the highway. The whole data set comprises measurements of about 780000 cars. By means of the detection devices it is possible to measure the passing time (up to a precision of $1 / 100$ sec), the speed of a vehicle, and the occupation rate of the loop. These direct measurements can be used in order to calculate the length of a vehicle, spatial and temporal distances between two cars and various other quantities of interest. The lower bound for the velocity measurement is $10 \mathrm{~km} / \mathrm{h}$, i.e., velocities of slower vehicles are not measured. The relation between direct measurements, and the spatial quantities as e.g.the distance headways are based on the assumption that the vehicles pass the detector at a constant and representative speed. This assumption is not valid for cars in a jam, where the detected speeds are much higher than the average speeds. 
As mentioned above the single-vehicle data allow for the determination of the time-headway $t^{h}$ and the distance-headway gap of the $n$-th vehicle via

$$
t^{h}(n)=t_{n}-t_{n-1}-\frac{l_{n-1}}{v_{n-1}}
$$

and

$$
g a p(n)=v_{n}\left(t_{n}-t_{n-1}\right)-l_{n-1}
$$

where we assume that $v_{n}$ and $v_{n-1}$ are constant. $t_{n}$ denotes the time the $n$-th vehicle passes the detector, $l_{n}$ and $v_{n}$ its length and velocity.

\section{$3.1 \quad t^{h}$ as a function of the velocity}

It is an obvious fact that the accident probability of a car largely depends on its distance to the vehicle in front. While the spatial headway strongly depends on the speed of the cars, it has been argued that the temporal headways are rather insensitive to the speed of the cars. Here we want to evaluate the velocity dependence of the time headway distribution. We have plotted the distribution for the $t^{h}$ of all vehicles having a velocity between $V$ and $V+\Delta V$ where $\Delta V=30$ $\mathrm{km} / \mathrm{h}$. Now, we take the value of $t^{h}$ for which the distribution is maximum $\left(t_{\max }^{h}\right)$, and plot it as a function of $V$. We choose the maximum $t_{\max }^{h}$ instead of the average value, because the average value is strongly influenced by the long tail for large $t^{h}$ 's - which is not of interest, as it reflects only the average flux. We rather expect the velocity to have an impact on the distribution at short headways. Our observation is that $t_{\max }^{h}$ is almost constant for all velocities greater than $80 \mathrm{~km} / \mathrm{h}$. Below this value, $t_{\max }^{h}$ increases as the velocity $V$ decreases. Drivers are impressed by driving too close (regarding spatial distance) to the preceeding car, while they are less sensitive to temporal distances.

\subsection{Identification of the platoons}

We define a platoon as a set of cars within which all time headways are less than a certain threshold $T_{\max }$, while the time headways in front and behind the platoon are larger than $T_{\max }$. This crude definition does not aim at characterizing a precise dynamical structure - some more specialized definitions of platoons have been given, which take into account for example the speeds of the cars, etc. Here, the extraction of the platoons from the data could rather be viewed as a pre-filtering of the data, on which the results should not depend.

\section{Impact of various security measures}

As the probability that a car brakes suddenly is not known, the absolute number of accidents we identify does not have any meaning. The relevant quantity is the relative number of accidents, when one compares two situations. Here, we use the result for the original data as a reference state. Then, we modify the data in 


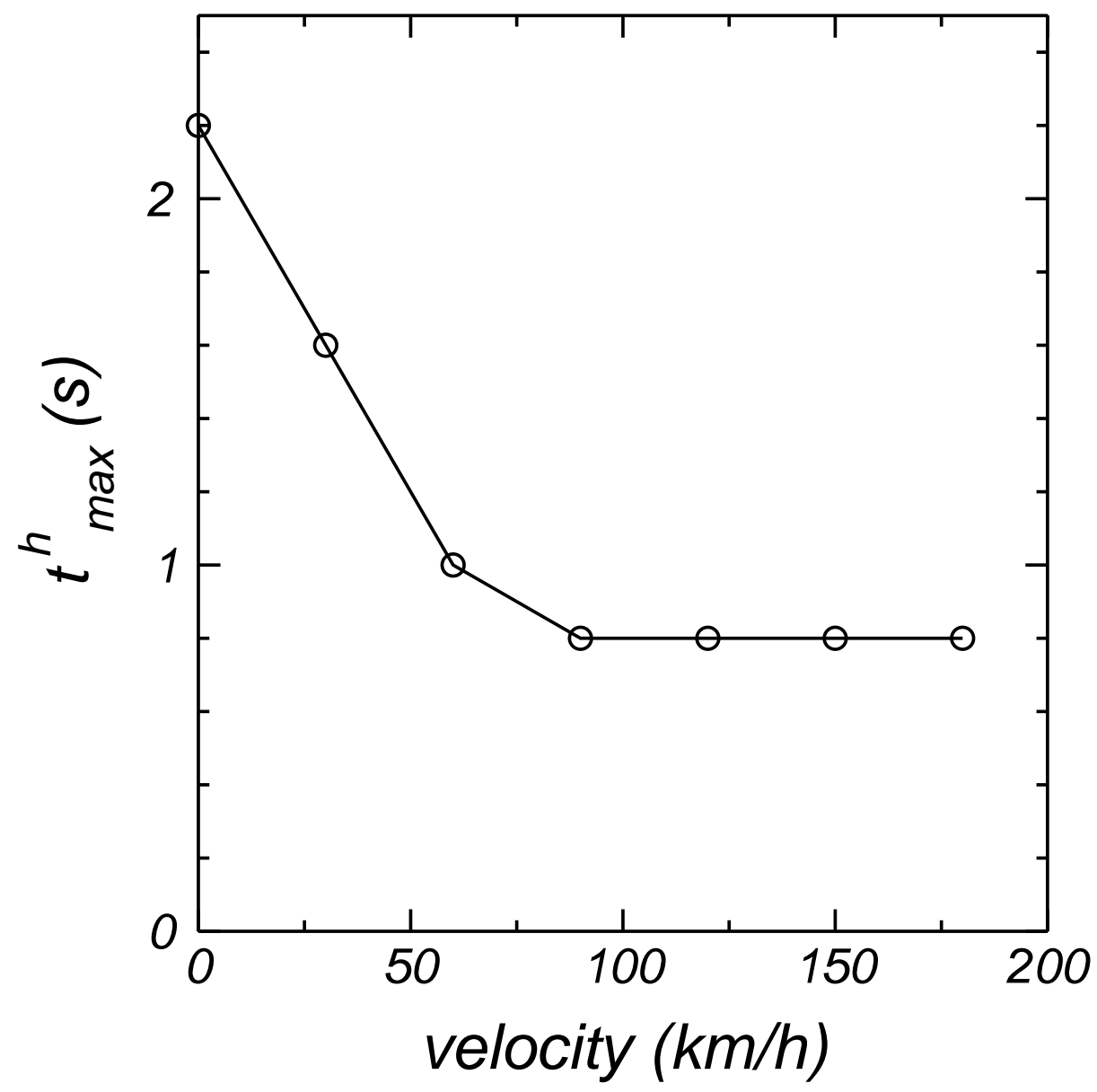

Fig. 2. $t_{\max }^{h}$ as a function of the velocity.

order to mimic various security measures. We compute the ratio of the number of accidents with a given security measure to the number of accidents in the reference state (see figure 3 ).

In a previous work [7], we have shown that our results depend only weakly on the choice for $T_{\max }$. Thus we present here only the results for $T_{\max }=3$ and $7 s$.

Now we study the dependence of the results with respect to the amplitude of the stimulus, i.e. the amplitude $a_{0}$ of the first braking car. The effects of the security measures (which are described below) are quite similar when the amplitude $a_{0}$ takes the values 3,4 , or $5 \mathrm{~m} / \mathrm{s}^{2}$.

The first possible security measure is the application of a speed limit, which we introduced in the following way: if the average velocity of a platoon $\bar{v}$ is above the speed limit $v_{\max }$, all the velocities within the platoon are rescaled by the factor $v_{\max } / \bar{v}$. In this way the relative speed dispersion is kept within the 
platoon. We also keep the time-headways between cars when applying the speed limit. This is justified by fig. 2, though it is possible that real speed limitations would have an impact on time-headways. But it is not obvious a priori in which direction the effect would be: one the one hand, more cars would drive at about the same speed, and thus there may be more competition between them. On the other hand, less drivers attempt to overtake as they have to respect the speed limit. Therefore the number of aggressive drivers should be reduced. A study by [1] shows that the fraction of drivers exceeding the speed limit $(130 \mathrm{~km} / \mathrm{h})$ is the same if you consider the subsets of vehicles with a time headway less than $0.5 \mathrm{~s}$ or 1.0s. This is in favor of a constant time headway for all velocities, at least for velocities above a certain threshold (around $80 \mathrm{~km} / \mathrm{h}$ in our case).

Fig. 3 shows that, counterintuitively, a speed limit increases the number of accidents. This is a consequence of the non-linearity of the braking trajectories, as explained in [7]. Of course, for most other accident scenarii, an increase of the speed would be a serious drawback.

The next points in figure 3 refer to a modification of the braking capacity distribution. As expected, an increase (decrease) of the width of the distribution increases (decreases) the number of accidents.

The first significant improvement on the number of accidents is obtained when the average of the braking capacity distribution is increased from 7 to $9 \mathrm{~m} / \mathrm{s}^{2}$. The effect is however limited, and in practice, it would be rather impossible to improve the braking capacities of all cars without increasing the width of the distribution.

The suppression of short time headways is by far the most efficient measure. If all time headways smaller than $1.8 \mathrm{~s}$ are replaced by time headways equal to 1.8 , almost all collisions are suppressed. Interestingly, even the suppression of time headways below $1 s$ only reduces the number of accidents drastically - more than half of the accidents are avoided.

\section{$5 \quad$ Perspectives}

A further improvement of the model should address the possibility that a vehicle reacts not only to the vehicle just in front, but also to other preceeding vehicles. This was already addressed in $[8,9]$ and recent work by S. Hoogendoorn [10] indicates such an influence of more than one preceeding car. When a car-following model is considered, it is easy to give a different weight - or sensibility - to the interaction depending on which pair of vehicles is considered. The results by S. Hoogendoorn indicate that if $\alpha_{0}$ is the sensibility for nearest neighbors, then a sensibility of the order of $\alpha_{0} / 2$ should typically be taken for next nearest neighbors.

In our case, which considers emergency braking, such a ponderation is not possible. Either one brakes or one does not brake. Our proposal would be that, though the action of braking would still follow the signal of the immediately preceeding car, preceeding cars could have an influence on the reaction time 


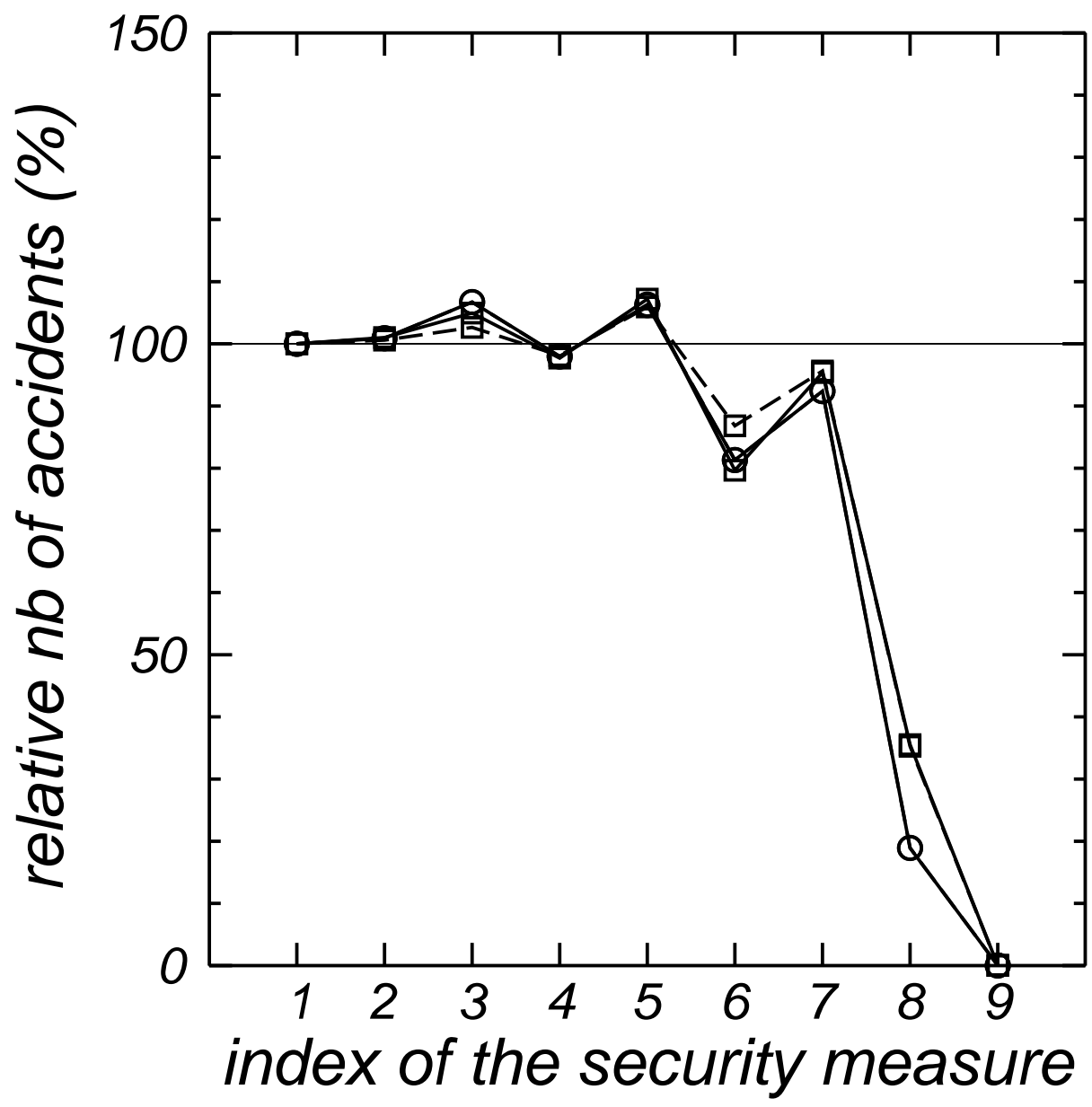

Fig. 3. Ratio of the number of accidents for various security measures. The 'x'-axis refers to these measures with the following correspondence: (1) is the reference state - by definition it is $100 \%$. (2) Speed limitation $v_{\max }=130 \mathrm{~km} / \mathrm{h}$. (3) $v_{\max }=110 \mathrm{~km} / \mathrm{h}$. (4) Braking capacities $a^{*} \in[6.8,7.2]\left(\mathrm{m} / \mathrm{s}^{2}\right)$. (5) $a^{*} \in[5,9]\left(\mathrm{m} / \mathrm{s}^{2}\right)$. (6) $a^{*} \in[8,10]\left(\mathrm{m} / \mathrm{s}^{2}\right)$. (7) Time headway minimum $t^{h} \geq 0.5$. (8) $t^{h} \geq 1.0$. (9) $t^{h} \geq 1.8$. Symbols circles, squares, x correspond respectively to an amplitude $a_{0}=3,4$, and $5 \mathrm{~m} / \mathrm{s}^{2}$. Solid (dashed) lines indicate that $T_{\max }=3 s(7 s)$.

distribution. I.e. if the driver observes that the 2nd car in front is braking, he prepares himself to react more rapidly.

One could also take advantage of the fact that in our data, the type of the vehicles is known (cars, trucks...). One could thus incorporate the fact that for example, a car behind a truck can only react on the vehicle directly in front, while other configurations allow for a larger visibility. 


\section{Conclusion}

We have shown that if something unexpected occurs on the road, as many drivers drive with a short headway, it is likely that they won't be able to avoid a collision. This suggests three possible levels of action to minimize the number of accidents:

- Decrease the number of unexpected events: this could be obtained for example by regularizing the flow, inciting the drivers to have more homogeneous velocities, etc.

- Suppress short time-headways: the equipment of cars with automatic distance control could be helpful.

- Improve reaction times: cars could be equipped with alert devices, etc.

Acknowledgments: LS acknowledges support by the Deutsche Forschungsgemeinschaft under Grant No. SA864/2-2. The authors are grateful to the Landesbetrieb Straßenbau NRW for providing the empirical data.

\section{References}

1. M. Aron, M.-B. Biecheler, and J.-F. Peytavin. Sécurité routière - temps intervéhiculaires et vitesse. quels enjeux de sécurité sur l'autoroute? Recherche Transports Sécurité, 64:3-17, 1999.

2. M. Brackstone, M. McDonald, and B. Sultan. A collision model for the assessment of the safety benefits of avcss. Proc. of the 6th ITS World Congress, Toronto, Canada., Nov., 1999.

3. J. Carbaugh, D.N. Godbole, and R. Sengupta. Safety and capacity analysis of automated and manual highway systems. Transportation Research Part C, 6:6999, 1998.

4. Rodger J. Koppa. Human factors. In Traffic Flow Theory, pages 3-1, 2000.

5. Des progrès pour la sécurité - l'automobile citoyenne. Les dossiers du CCFA.

6. R.J. Kiefer, M.T. Cassar, C.A. Flanagan, C.J. Jerome, and M.D. Palmer. Surprise braking trials, time-to-collision judgments and "first look" maneuvers under realistic rear-end crash scenarios. In DC Contract DTFH61-01-X-00014, Washington, editor, Performed by Crash Avoidance Metrics Partnership (CAMP), August 2005.

7. C. Appert and L. Santen. Accidents in dense vehicular traffic. preprint, 2005.

8. H. Lenz, C.K. Wagner, and R. Sollacher. Multi-anticipative car-following model. The European Physical Journal B - Condensed Matter, 7:331-335, 1999.

9. M. Treiber, A. Kesting, and D. Helbing. Delays, inaccuracies and anticipation in microscopic traffic models. Physica A, 360:71-88, 2006.

10. S.P. Hoogendoorn, S. Ossen, and M. Schreuder. Multi-anticipative car-following behavior: and empirical analysis. preprint, 2005. 